\title{
High-Speed Target Identification System Based on the Plume's Spectral Distribution
}

\author{
Wenjie Lang, ${ }^{1}$ Guoguang Chen, ${ }^{2}$ Xiaoli Tian, ${ }^{2}$ and Changfan Xin ${ }^{2}$ \\ ${ }^{1}$ School of Information and Communication Engineering, North University of China, Taiyuan, Shanxi 030051, China \\ ${ }^{2}$ School of Mechatronics Engineering, North University of China, Taiyuan, Shanxi 030051, China \\ Correspondence should be addressed to Wenjie Lang; langwenj@163.com
}

Received 15 September 2015; Revised 12 November 2015; Accepted 15 November 2015

Academic Editor: Yu Shang

Copyright (c) 2015 Wenjie Lang et al. This is an open access article distributed under the Creative Commons Attribution License, which permits unrestricted use, distribution, and reproduction in any medium, provided the original work is properly cited.

\begin{abstract}
In order to recognize the target of high speed quickly and accurately, an identification system was designed based on analysis of the distribution characteristics of the plume spectrum. In the system, the target was aligned with visible light tracking module, and the spectral analysis of the target's plume radiation was achieved by interference module. The distinguishing factor recognition algorithm was designed on basis of ratio of multifeature band peaks and valley mean values. Effective recognition of the high speed moving target could be achieved after partition of the active region and the influence of target motion on spectral acquisition was analyzed. In the experiment the small rocket combustion was used as the target. The spectral detection experiment was conducted at different speeds $2.0 \mathrm{~km}$ away from the detection system. Experimental results showed that spectral distribution had significant spectral offset in the same sampling period for the target with different speeds, but the spectral distribution was basically consistent. Through calculation of the inclusion relationship between distinguishing factor and distinction interval of the peak value and the valley value at the corresponding wave-bands, effective identification of target could be achieved.
\end{abstract}

\section{Introduction}

The rockets are nonguided munitions whose motor ability is formed by rocket engine. Their subtypes are mainly pickaback rockets and vehicle multiple mounted rockets [1]. They are mainly used to kill, repress, and blow up enemies' armed fortifications and light armor troops, and so forth. Compared with artillery, their mobility, rapid reaction capacity, and the widespread destruction ability are outstanding $[2,3]$. When they are in comparison with laser-guided bombs and cruise missiles, they are much cheaper although their accuracy and lethality are relatively inferior. Therefore although rockets were invented at the beginning of 19th century, they are still used in the battlefield all over the world. All the military powers in the world led by the United States have been developing the new rocket system such as AT4 modification.

The effective identification and rapid response of the rocket is the important guarantee for the survival of the wartime army, so it is significant to study the detection system of the rocket type, velocity, and killing area. Identification method for target of high speed attack includes radar detection [4], image recognition [5], and spectral recognition [69]. Radar detection technology has a wide detection range and high signal stability, but it is obviously influenced by the surface environment. Image recognition can constitute the target infrared image with high temperature characteristics of rocket plume flame, which can realize long-distance passive detection with high concealment but cannot identify the target type and can easily be affected by other rounds of fire in the battlefield. Spectral identification can identify rocket's type and velocity based on analysis of spectral intensity and distribution characteristic of rocket plume. It has the characteristics of fast detection speed, good concealment, and vulnerable to the impact of the fire flash in the environment. But in the traditional method, it is necessary to obtain the spectral distribution characteristics of the target previously so as to achieve the target identification. In addition, the target speed and other parameters need to be assisted by other detection systems. 


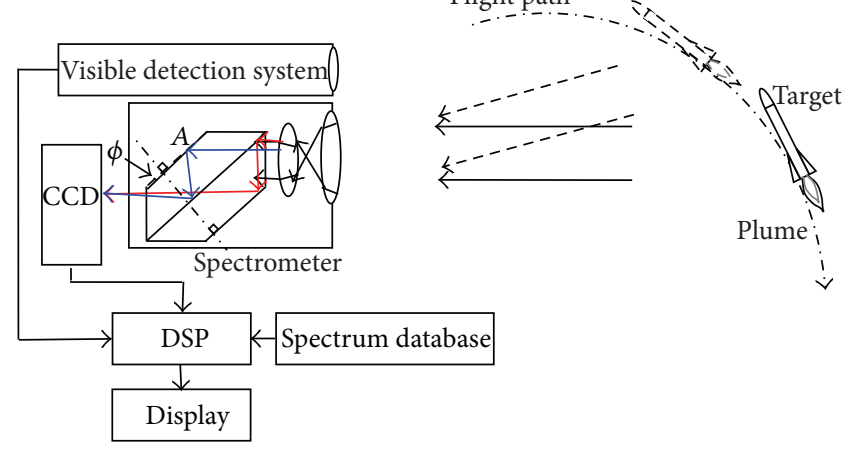

FIGURE 1: Principle of location identification system based on target's plume.

The main content of this paper is the spectral identification system based on the rocket plume. The purpose is to analyze and process the important parameters, such as the type and speed of the target type, hence improving the identification ability of the target of rocket projectile by the design of spectral data processing algorithm.

\section{Design of High Speed Target Identification System}

Figure 1 shows that when traversing through the system's detection area along the flight path, the target was divided into two parts. One was visible light target tracking system, which could complete the coarse positioning of the target to ensure that the spectral acquisition system could aim at the target effectively. Another was spectral acquisition system which combined space interference module with CCD, the charge-coupled device. Space interference module refers to the static interferometer structure, that is, processing a 0.1 degree dip angle on the reflection side to produce the effect of space scanning. The effective length of the interferometer is $50.0 \mathrm{~mm}$. This method avoided the detection error caused by mechanical scanning, and it was more suitable to do field work. The interference fringe data only needed to be collected once, greatly reducing the time of spectral data acquisition and achieving real-time processing of high speed target. The spectral distribution of the target tail flame was obtained by the interference system, whose spectral intensity was significantly higher than that of the background spectrum, and it had different spectral characteristics according to different missile types, so that the target could be accurately retrieved. As the system detects the target of high speed flight, it is not necessary to obtain the background spectrum data in advance. However, the target distance can affect the spectral energy, so it is necessary to analyze and calculate the spectral distribution characteristics by solving the target type through proportional computation or feature algorithm. By analyzing the spectral changes and spectral shape of the target, this system can determine the type and other types of information of the target; thus the target has high light flux and a stable high speed.

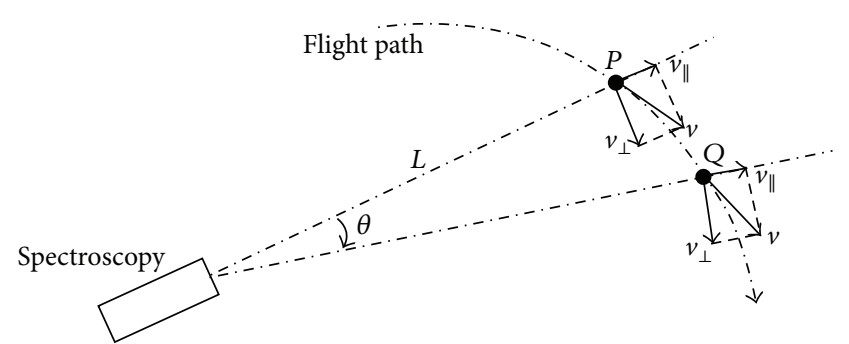

FIGURE 2: Decomposition of target motion characteristics.

\section{Effect of Target High Speed Motion on Spectral Recognition}

3.1. Spectral Acquisition. The photometry entering the system interference module was divided into two optical beams of equal intensity by the transflective film. Set the light intensity as $I_{0}$. When the two optical beams' coherence is on the photosensitive surface of CCD (charge-coupled device), their intensity distribution can be expressed as

$$
I=2 I_{0}[1+\cos (2 \pi v l)]
$$

where $v$ is wave number $\left(v=\lambda^{-1}\right)$ and $l$ is the distance from the measured point to the zero optical path in interference module. By Fourier transform with the interference data [10], the spectral distribution function of infrared radiation can be determined as in the following formula:

$$
E(v)=\int_{-\infty}^{+\infty} I(l) e^{-i 2 \pi v l} d l
$$

The spectral distribution function of the target's tail flame can be determined by the above formula, so that the identification of the target species based on spectral distribution characteristics is obtained. The interference fringes obtained by the system are formed by the spatial scanning. The spectral distribution data of the target can be obtained with smooth filtering, inversion, FFT (fast Fourier transform), and spectral calibration of the interference fringes.

3.2. Generation of Spectral Offset. The rapid identification of the remote and disguising stationary maneuvering target has been studied by spectral analysis technology. The working process is to compare the difference between measured target spectral distribution and background spectral distribution and then obtain the information of the target.

For both active light source and passive radiation measurement, the target and detection system in the testing process are static so as to constitute a stable test mode. However, for high-speed moving target, the echo spectrum cannot be simply extracted directly, because the high speed moving target can produce the corresponding spectral shift effect in the process of spectral acquisition.

As shown in Figure 2, because it is impossible for the visible light optical target tracking system to enable optical interference system realizing real-time targeting, the real physical process is that, in a calibration cycle (or tracking 
cycle), the spectral information gathered by optical interference system is the changed spectral information from point $P$ to point $Q$. The time from point $P$ to $Q$ equals the calibration period of visible light tracking system aiming at system collimation direction. Although the distance between the target and the system ranges from dozens to hundreds of kilometers and tracking and calibrating time is very short, namely, $\theta$ angle is a very small, its impact on the characteristics of spectral distribution is very significant.

First, the $P$-point instantaneous velocity is decomposed into $\left.v_{\|}\right|_{P}$ and $\left.\left.v_{\perp}\right|_{P} \cdot v_{\|}\right|_{P}$ means that the velocity is parallel to the optical axis of optical interference system while $\left.v_{\perp}\right|_{P}$ refers to the fact that the velocity is in vertical direction. At the same time Q-point instantaneous velocity is decomposed into $\left.v_{\|}\right|_{Q}$ and $\left.\left.v_{\perp}\right|_{Q} \cdot v_{\|}\right|_{Q}$ and $\left.v_{\perp}\right|_{Q}$ also mean that the velocity is parallel and is vertical to optical axis of optical interference system.

(1) For $v_{\perp}$, light source has a reverse speed at the detector, resulting in the Doppler Effect. The detector's reception frequency $v_{T}$ is calculated with the following formula based on Doppler effect [11]:

$$
v_{T}=v_{0} \sqrt{\frac{c-v_{\perp}}{c+v_{\perp}}},
$$

where $v_{0}$ is the target's light frequency and $c$ is the light speed. The speed of the conventional rocket is about 3-6 Mach (1 Mach $=340 \mathrm{~m} / \mathrm{s}$ ), whereas the light speed is $3.0 \times 10^{8} \mathrm{~m} / \mathrm{s}$. In theory, even though a factor about $3.4 \times 10^{-6} \sim 1.2 \times 10^{-5}$ (calculated with Doppler Effect Formula) is used to correct spectral frequency in its radial movement, the impact on the spectral distribution is smaller.

(2) For $v_{\|}$, in a calibration cycle, the light source moves from $P$-point to $Q$-point, which equals the tiny displacement of the incident angle. This displacement is decided by the speed of the target and the sample period. When the geometrical relation between the target and detector is considered,

$$
\theta \approx \operatorname{arctg}\left(\frac{P Q}{L}\right) \approx \operatorname{arctg}\left(\frac{v_{t} T}{L}\right),
$$

where $v_{t}$ is velocity; $T$ is sampling calibration period; $L$ is the distance from the target to the detector. Suppose that the rocket comes into the detection area at velocity of 3.5 Mach; its update time is to $100 \mathrm{~ms}$ and the detection distance is to $100 \mathrm{~km}$; it can generate the offset angle $\theta$ as 0.068 degree. Calculation shows that when the effective size of interference mould is $50.0 \mathrm{~mm}$, its optical path variation is $59.5 \mu \mathrm{m}$, thus causing more obvious spectral offset. In this experiment target at $2 \mathrm{~km}$ distance is used to test the feasibility of the method. If target at $2 \mathrm{~km}$ could be measured, as for target at distance of $100 \mathrm{~km}$, the effective detector could be achieved when the target radiation intensity is sufficient enough and the spectral resolution of the system's static interferometer can be further increased.

\section{Experiments}

4.1. Experiment Condition. In laboratory the analysis of spectral data should be conducted with simulation experiment.
Select a small amount of rocket burning part to do the spectrum test, and analyze echo spectrum of the high-speed moving target. In combination with the ratio of real target radiation energy and detection distance, the simulation test approximates the result by proportionally scaling the physical process. As the radiation intensity of the target depends mainly on the distance from the target to the detector $\left(I \propto d^{-2}\right)$, the simulated target is placed at $2.0 \mathrm{~km}$ from the detector based on the actual situation of the test field, the plume burning energy value, and the relation between intensity and distance. In order to obtain the impact degree of target radiation on the spectrum detection under different velocities, we made the actual speed of the test target to be approximately $676 \mathrm{~m} / \mathrm{s}, 1009 \mathrm{~m} / \mathrm{s}, 1354 \mathrm{~m} / \mathrm{s}$, and $1678 \mathrm{~m} / \mathrm{s}$ (selection basis is that target's velocity can cover roughly 2-5 Mach) to observe their effects on echo spectral data.

4.2. Experimental Data and Analysis. In the experiment, the instrument is constituted by static interference prism with effective length of $50.0 \mathrm{~mm}$. The interference fringes are acquired by the interference system, and the interference fringes are converged to a one-dimensional image through a cylindrical lens, which will be received by InGaAs linear CCD (charge-coupled device) of HONEYWELL Company. At the position of $2.0 \mathrm{~km}$, when the simulation target (the small amount of rocket burning part) moves tangentially at speed of $676 \mathrm{~m} / \mathrm{s}, 1009 \mathrm{~m} / \mathrm{s}, 1354 \mathrm{~m} / \mathrm{s}$, and $1678 \mathrm{~m} / \mathrm{s}$, respectively, its spectral data constitute the spectral distribution as shown in Figures 3(a), 3(b), 3(c) and 3(d).

Figure 3 indicates that for the same simulation target, its spectral distribution is similar in shape. The appropriate characteristic wavelength position is obtained by spectral data processing algorithm, from which the proportional relationship of the characteristic wavelength could be used to deduce the target species. As shown in the figure, there is peak value, but it is not very prominent. However, since the system itself does not judge the target species by its peak value but by the ratio of peak to valley in the subregion of the target and the data in the known spectral database, whether the speed is changed or not, the peak jitter is just relative to the original spectrum, thus having not much effect on the test results.

When the simulation target speed is $676 \mathrm{~m} / \mathrm{s}$, spectral maximum value position is at $1422 \mathrm{~nm}$. With the increase of speed, the spectral distributions obtained by Fourier transform shift accordingly. Finally, when the target speed is $1678 \mathrm{~m} / \mathrm{s}$, the maximum spectrum position is at $1432 \mathrm{~nm}$. The simulation target velocity is much slower than the speed of light, so the red shift of the optical Doppler Effect is unlikely to produce such a large spectral offset. Therefore, the main source of spectral offset stems from the offset of incident radiation angle formed by target tangential motion within a sampling calibration cycle.

Although the target spectrum is shifted and the amplitude is slightly changed, the spectral distribution pattern is basically consistent, and the characteristic wavelength position can be used to solve the spectrum distribution. The feasibility to measure high speed target types based on characteristic spectral distribution is proved. 


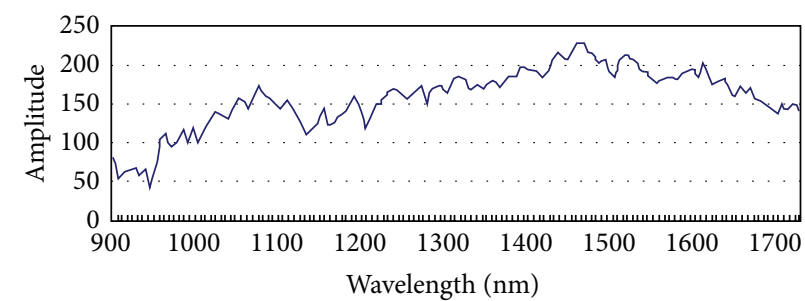

(a)

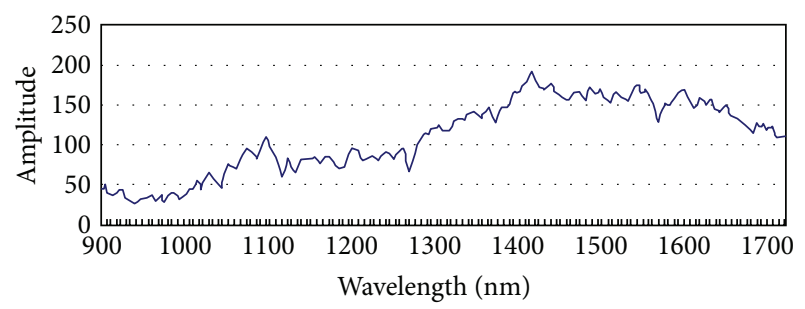

(c)



(b)

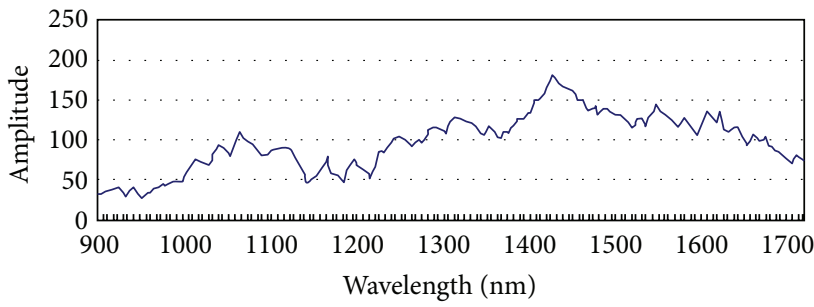

(d)

FIGURE 3: Characteristic spectrum of stationary targets and moving targets.

At the same time, it also puts forward that the detection process needs to meet the constraints; that is, the target needs to have quicker tangential speed and a certain sampling calibration cycle (accumulated time).

4.3. Process of Characteristic Spectral Data. On the basis of the above analysis, the spectral analysis algorithm is needed to identify the target specie. Because this system can refer to the parameters in the spectrum database as the basis, it is different from other common spectral analysis algorithms. By using the selective region, the subregion and the wavelength number are set up by the test requirement, and the target can be effectively identified by the ratio of different peak and valley, which can greatly improve the recognition efficiency of the system. Because the amplitude and spectral shift of the target high speed motion are obvious, even though the target can roughly maintain the original spectral shape, the characteristic wavelength position should be in accordance with the spectral ratio of the characteristic wavelength, so as to ensure the effective selection of the characteristic wavelength. The following are the steps of value ratio algorithm:

(1) $M$ subregions are set up in the whole range of detection wavelength, and the selection principle of $M$ is determined by the size of the detection range and the location of the characteristic peak.

(2) In each subregion the selection of several characteristic peak wavelength position contains $\lambda_{1}, \lambda_{2}, \ldots, \lambda_{n}$ to constitute a characteristic wavelength group. Take the mean value of the mean $\left(\bar{I}_{n}\right)$ as the peak value of $A$ group. In the same way, a set of wavelengths (the number is consistent with the feature group) is selected, which is the smallest of the spectral amplitude in the subregion. Take the arithmetic average value as the valley mean value of $B$ group.
(3) The ratio of $A$ group and $B$ group is taken as a discriminating factor $\sigma_{i}$. Then the classification algorithm is used to classify the attributes according to the distribution of the different factors. The distribution intervals of distribution factors are affected by detector species and the spectral overlap of different targets. In the experiment, due to the less number of species being distinguished, when $m=3, n=5$, the distinction interval $\left[0.9 \sigma_{i}, 1.1 \sigma_{i}\right]$ can meet the testing requirements.

The spectrum of target 1 , interference targets 2 and 3 , the flame noise, and the sun light noise were identified. The target refers to the one that is required to be identified in the system, while the interference target means those who do not need identifying, but their radiation energy is similar to that of the target in the detection field. Flame noise refers to the interference caused by the explosion, flame, and so forth in the field. The sun light noise means the interference of the solar radiation in the test. The peak value of $A$ and $B$, the ratio of the measured object, and the recognition probability are given, as shown in Table 1.

As shown in Table 1, we can obtain the peak values $A$ and $B$ of each subregion by solving the three subregions and then obtain the value of the ratio. Since target 1 is seen as the test object, the peak group and the proportion coefficient should be considered by referring to the spectrum selection of target 1 , thus producing the corresponding proportion coefficient of the interference targets 2 and 3, fire flame, and sun light flame. Since the distinguished interval in the system is designed as $\left[0.9 \sigma_{i}, 1.1 \sigma_{i}\right]$, the ranges of the three characteristic bands are $1.926 \sim 2.354,1.548 \sim 1.892$, and 1.008 1.232. That means that when the distinguished factor of the measured spectral data is within this range, the target cannot be distinguished. The target can only be recognized only when its distinguishing factor is within this interval. It shows that target 1 can be identified by the characteristic wavelength in region 1 , while 
TABLE 1: Mean peak value and proportional coefficient of spectral characteristic of measured target and distracters and background noise.

\begin{tabular}{|c|c|c|c|c|c|c|c|c|c|}
\hline & \multicolumn{3}{|c|}{ Characteristic bands 1} & \multicolumn{3}{|c|}{ Characteristic bands 2} & \multicolumn{3}{|c|}{ Characteristic bands 3} \\
\hline & $\begin{array}{c}\text { Mean peak } \\
A\end{array}$ & $\begin{array}{c}\text { Mean peak } \\
B\end{array}$ & Ratio value & $\begin{array}{c}\text { Mean peak } \\
A\end{array}$ & $\begin{array}{c}\text { Mean peak } \\
B\end{array}$ & Ratio value & $\begin{array}{c}\text { Mean peak } \\
A\end{array}$ & $\begin{array}{c}\text { Mean peak } \\
B\end{array}$ & Ratio value \\
\hline Target 1 & 162 & 79 & 2.14 & 213 & 124 & 1.72 & 194 & 175 & 1.12 \\
\hline Target 2 & 85 & 93 & 0.91 & 187 & 166 & 1.13 & 106 & 97 & 1.09 \\
\hline Target 3 & 186 & 114 & 1.63 & 115 & 134 & 0.86 & 238 & 187 & 1.27 \\
\hline Flame & 67 & 58 & 1.16 & 206 & 126 & 1.63 & 75 & 48 & 1.56 \\
\hline Solar & 84 & 73 & 1.15 & 66 & 57 & 1.16 & 61 & 52 & 1.17 \\
\hline \multicolumn{2}{|c|}{ Distinguish range } & \multicolumn{2}{|c|}{$1.926 \sim 2.354$} & \multicolumn{3}{|c|}{$1.548 \sim 1.892$} & & \multicolumn{2}{|c|}{$1.008 \sim 1.232$} \\
\hline \multicolumn{3}{|c|}{ Whether there is recognition } & Yes & & & No & & & No \\
\hline
\end{tabular}

those in regions 2 and 3 cannot be identified. Even when there are two or more distinguishing factors within the interval in these three regions, the target's effective recognition could still be done through the analysis whether the interference target's spectrum is in accordance with its distinguishing interval position or not or through the calculation of the ratio of interference target's spectral distinguishing factors.

\section{Conclusion}

In this paper, a recognition system is designed based on the analysis of the plume spectral distribution. The traditional target recognition system based on spectral analysis is applied to the target of high speed, such as rocket, and other weapons. The main effects of the high speed moving target on the spectral inversion are analyzed by theoretical derivation and the correlation function is given. In the experiment, the spectral distribution of the target under different speed conditions is analyzed with a small amount of rocket fire. The recognition of target can be achieved under the condition of spectral shift. And by means of the difference between the peak value and the mean value of the valley in the multibands, the method of identifying the characteristic spectral proportion is formed. In addition, the function of the effective recognition of the target is realized through the analysis of the corresponding relationship between the different factors and intervals.

\section{Conflict of Interests}

The authors declare that there is no conflict of interests regarding the publication of this paper.

\section{Acknowledgments}

This work was supported by the National Natural Science Foundation of China (no. 51305409) and Army Academy longitudinal Project (no. JZ2009100).

\section{References}

[1] M. Iodice, V. Striano, and G. Cappuccino, "Fiber bragg grating sensors based system for strain measurements," in Proceedings of 4th IEEE/LEOS Workshop on Fibres and Optical Passive Components (WFOPC '05), pp. 307-312, IEEE, June 2005.

[2] E. Acosta, S. Chamadoira, and R. Blendowske, "Modified point diffraction interferometer for inspection and evaluation of ophthalmic components," Journal of the Optical Society of America: Optics and Image Science, and Vision, vol. 23, no. 3, pp. 632-637, 2006.

[3] A. K. Sharma, "Fiber-optic sensors based on surface plasmon resonance: a comprehensive review," IEEE Sensors Journal, vol. 7, no. 8, pp. 1118-1129, 2007.

[4] Y. Zhang, Y.-F. Shen, J. Zhou et al., "Positive-negative birefraction phenomenon for TM polarization in annular photonic crystal," Chinese Optics Letters, vol. 9, no. 2, pp. 81-86, 2011 (Chinese).

[5] K. Sun, X.-G. Sun, and J.-M. Dai, "Development of multispectral thermometer for explosion flame true temperature measurement," Spectroscopy and Spectral Analysis, vol. 31, no. 3, pp. 849-852, 2011 (Chinese).

[6] D. J. Poxson, F. W. Mont, M. F. Schubert, J. K. Kim, J. Cho, and E. F. Schubert, "Demonstration of optical interference filters utilizing tunable refractive index layers," Optics Express, vol. 18, no. 23, pp. A594-A599, 2010.

[7] J. S. Melinger, N. Laman, and D. Grischkowsky, “The underlying terahertz vibrational spectrum of explosives solids," Applied Physics Letters, vol. 93, no. 1, Article ID 011102, 2008.

[8] N. Laman, S. S. Harsha, D. Grischkowsky, and J. S. Melinger, "7 $\mathrm{GHz}$ resolution waveguide $\mathrm{THz}$ spectroscopy of explosives related solids showing new features," Optics Express, vol. 16, no. 6, pp. 4094-4105, 2008.

[9] J. Li, J.-P. Zhu, Y.-Y. Zhang, H. Liu, and X. Hou, "Spectral zooming birefringent imaging spectrometer," Acta Physica Sinica, vol. 62, no. 2, Article ID 024205, 2013.

[10] P. G. Lucey, K. A. Horton, and T. Williams, "High-performance Sagnac interferometer using cooled detectors for infrared LWIR hyperspectral imaging," in Airborne Intelligence, Surveillance, Reconnaissance (ISR) Systems and Applications IV, B. Denevi, Ed., vol. 6546 of Proceedings of SPIE, p. 7, 2007.

[11] T. Lin, F. Liu, P. Han et al., "Band selection method of spectrum detection about medium wave infrared stealth target," Infrared and Laser Engineering, vol. 43, no. 4, pp. 1047-1051, 2014 (Chinese). 

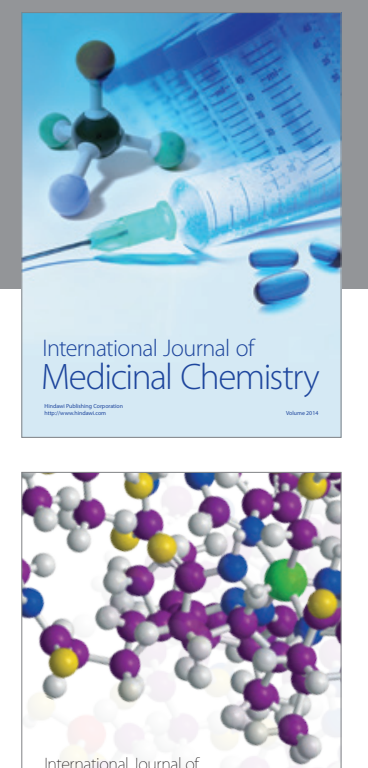

\section{Carbohydrate} Chemistry

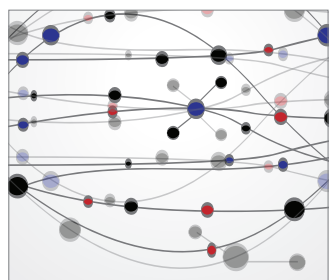

The Scientific World Journal
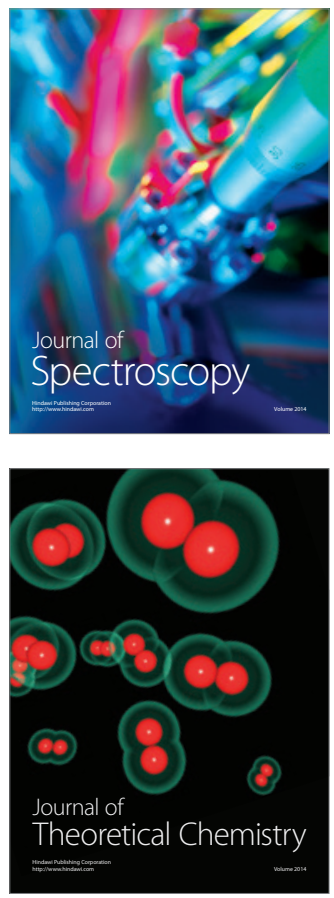
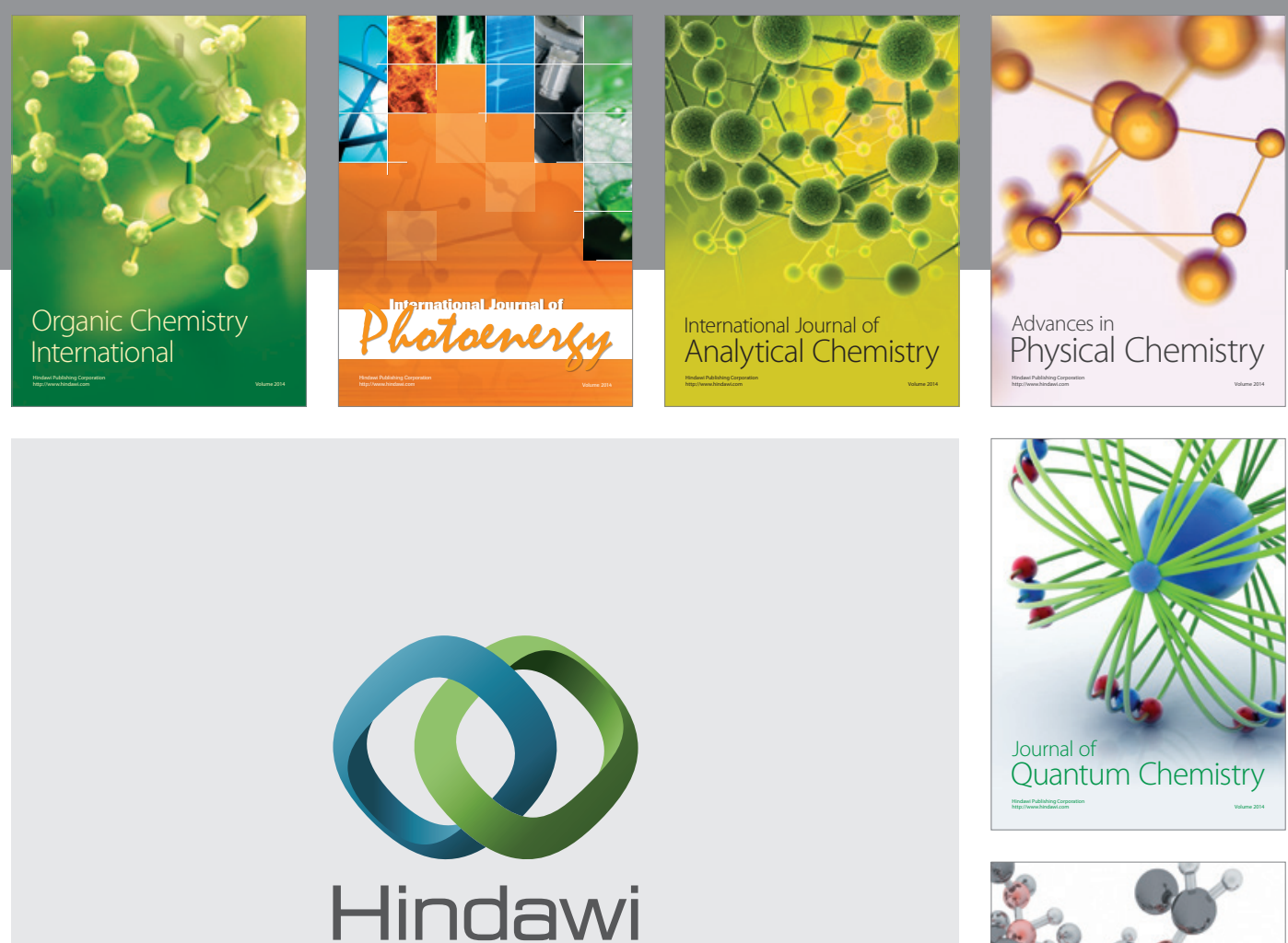

Submit your manuscripts at

http://www.hindawi.com



Analytical Methods

in Chemistry

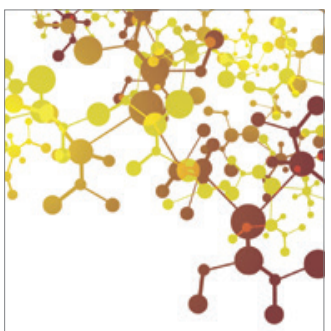

Journal of

Applied Chemistry

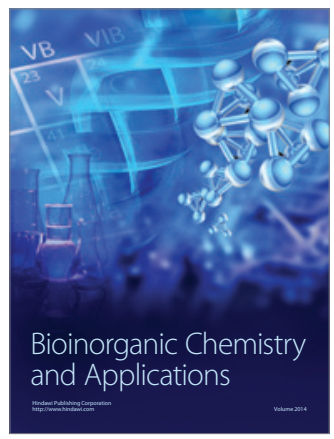

Inorganic Chemistry
